\title{
Multiple Antibiotic Resistance Plasmids Allow Scalable, PCR-Mediated DNA Manipulation and Near-Zero Background Cloning
}

\author{
Remigiusz Arnak, Burcin Altun, Valentina Tosato and Carlo V. Bruschi* \\ Yeast Molecular Genetics Laboratory, ICGEB, AREA Science Park, Padriciano 99, IT-34149 Trieste, Italy
}

Received: April 23, 2015

Accepted: February 26, 2016

\begin{abstract}
Summary
We have constructed two plasmids that can be used for cloning as templates for PCR-based gene disruption, mutagenesis and the construction of DNA chromosome translocation cassettes. To our knowledge, these plasmids are the first vectors that confer resistance to ampicillin, kanamycin and hygromycin B in bacteria, and to geneticin (G418) and hygromycin B in Saccharomyces cerevisiae simultaneously. The option of simultaneously using up to three resistance markers provides a highly stringent control of recombinant selection and the almost complete elimination of background resistance, while unique restriction sites allow easy cloning of chosen genetic material. Moreover, we successfully used these new vectors as PCR templates for the induction of chromosome translocation in budding yeast by the bridge-induced translocation system. Cells in which translocation was induced carried chromosomal rearrangements as expected and exhibited resistance to both, G418 and hygromycin B. These features make our constructs very handy tools for many molecular biology applications.
\end{abstract}

Key words: DNA manipulation, multiple-antibiotic resistance, multi-marker vectors, gene knock-out, functional analysis, yeast

\section{Introduction}

Plasmid-mediated antibiotic resistance is commonly used as selection criterion for genetically engineered bacteria and fungi due to its efficiency and the availability of the respective drugs from commercial sources $(1,2)$. For instance, many functional and genetic studies of yeast and other organisms require convenient plasmid templates for PCR-mediated gene disruptions, deletions (3), the recently developed bridge-induced translocation (BIT) system $(4,5)$ and for many other purposes.

Combining two or three resistance genes simultaneously on the same DNA vector can facilitate selection and eliminate false positives (spontaneous acquisition of resistance to three different drugs is rather improbable, excluding resistance phenotypes caused by the elevated efflux of drugs from the cell) $(2,6)$. Moreover, a strict selection in combination with stable maintenance will be very advantageous when using shuttle plasmids as shuttle vectors for cloning purposes in yeast. For this reason, we decided to develop a plasmid that is highly selective for both expression purposes as well as for the preparation of functional analysis constructs, allowing a substantial decrease in unwanted background resistance. This has been achieved by combining the coding sequences for antibiotic resistance to three widely available drugs: ampicillin, kanamycin (geneticin in yeast) and hygromycin B, in a specially engineered order. The result was the construction of two plasmids (pMM and pMM-CEN) conferring simultaneous resistance to these drugs, while lending themselves to be easily manipulated and utilized as vectors. As an experimental application of these new vectors, the pMM plasmid was employed as PCR template in the BIT process (5). BIT utilizes homologous recombina- 
tion to physically bridge two loci of choice within the yeast genome, with a double-stranded DNA amplicon (cassette) amplified from a pMM template, in which a positively selectable marker is flanked by short regions of homology (typically $65 \mathrm{bp}$ ) to the two loci of choice.

\section{Materials and Methods}

\section{Bacteria and yeast strains}

Escherichia coli XL1-Blue (catalog \#200249; Stratagene, Santa Clara, CA, USA), $\Delta(m c r A) 183 \Delta(m c r C B-h s d S M R-$ -mrr)173 endA1 supE44 thi-1 recA1 gyrA96 relA1 lac [ $F^{\prime}$ proAB lacl ${ }^{q} Z \Delta M 15$ Tn10 $\left(\right.$ Tet $\left.\left.^{r}\right)\right]$, E. coli Sure2 (catalog \#200152; Stratagene), endA1 glnV44 thi-1 gyrA96 relA1 lac recB recJ sbcC umuC::Tn5 uvrC e14- $\Delta$ (mcrCB-hsdSMR-mrr)171 $\mathrm{F}^{\prime}\left[\right.$ proAB $^{+}$lacl $^{q}$ lacZ $\Delta$ M15 Tn10 Amy $\left.\mathrm{Cm}^{R}\right]$ and E. coli DH5 $\alpha$ (catalog \#DA100; McLab, San Francisco, CA, USA), $F^{-}$endA1 glnV44 thi-1 recA1 relA1 gyrA96 deoR nup G Ф80dlacZ $\Delta M 15 \Delta\left(\right.$ lacZYA-argF)U169, $h s d R 17\left(r_{K}^{-} m_{K}{ }^{+}\right), \lambda$ were used in this work. Strains XL1-Blue and Sure2 were used routinely for plasmid propagation, cloning and plasmid construction because of their colour screening and improved cloning efficiency. Strain DH5 $\alpha$ is a more general type that was used as control of the efficiency of transformation. Luria Bertani (LB) broth for bacteria was prepared following standard protocols (7) and supplemented with $100 \mu \mathrm{g} / \mathrm{mL}$ of ampicillin, $100 \mu \mathrm{g} / \mathrm{mL}$ of kanamycin and/or $100 \mu \mathrm{g} / \mathrm{mL}$ of hygromycin B (Invitrogen, Carlsbad, CA, USA) as required. The Saccharomyces cerevisiae yeast strain SAN1 used in this work (MAT a/a ura3-52/ura3-52, lys2-80/+, ade1/+, ade2-10/ade2, ade8/+, trp1-A1/trp1-289, his3-A200/+, leu2-Al/leu2, can1/+, arg4:FRTG-NLS-tetR-GFP/ARG4::FRTG-DsRed) was previously developed in our laboratory (8). Yeast extract, peptone, dextrose (YPD; Difco, Sparks, MD, USA) supplemented with geneticin (G418) and/or hygromycin B (final concentration 200 and $300 \mu \mathrm{g} / \mathrm{mL}$, respectively) was used as a selective medium for yeast transformants as described previously (9).

\section{Plasmid construction}

All described constructs were built on the pFA6a-kanMX4 (accession number AJ002680; European Molecular Biology Laboratory, EMBL) plasmid platform (10). Firstly, the hygromycin B resistance gene (hphMX4) was amplified from plasmid pAG32 using PCR (11). The PCR product was designed in such a way that it contained a BamHI restriction site at the $5^{\prime}$ end, the $H y g^{R}$ gene, and the $K p n I-B g l I I$ site at the $3^{\prime}$ end of the amplicon. Primers used for construction, verification and testing are reported in Table 1.

All PCR reactions were carried out using Kapa HiFi polymerase (Kapa Biosystems, Woburn, MA, USA) following the manufacturer's instructions. The amplified fragments were purified afterwards using the Qiagen PCR product purification kit (Qiagen $\mathrm{GmbH}$, Hilden, Germany). Plasmid pFA6a-kanMX4 was linearized using BamHI-BglII (New England Biolabs, Ipswich, MA, USA) and hphMX4, a hygromycin B resistance gene ending with appropriate restriction sites, was ligated into the resulting gap using standard protocols (7). The DNA fragment was designed in such a way that it restored functional BamHI and BglII sites after ligation. The DNA construct was used to transform genetic recombination-deficient Sure2 and XL1-Blue E. coli strains to ensure the lack of recombination events between neighbouring DNA translational elongation factor (TEF) elements, and the strains were plated on plates containing either or both ampicillin and hygromycin B (in the case of strain Sure2), or on plates with one or more of the ampicillin, kanamycin and hygromycin B (for strain XL1-Blue). After one day of growth, plasmids were isolated from one of the two strains using the Promega mini-prep kit (Promega, Madison, WI, USA) and used to transform the other E. coli strain. The DNA construct was verified by PCR with primers specific for the kanamycin resistance, the hphMX4 and the combination of the two genes by restriction fragment length polymorphism (RFLP, using AatII, BglII, BamHI-BglII and MluI restriction enzymes) and finally validated by transforming E. coli $\mathrm{DH} 5 \alpha$ and selecting on LB agar plates containing kanamycin, hygromycin B and ampicillin (7). The resulting plasmid was named $\mathrm{pMM}$ and it contained genetic determinants conferring simultaneous resistance to ampicillin, kanamycin (geneticin for yeast) and hygromycin B. Between the kanamycin and hphMX4 resistance genes there is a preferential cloning site that allows the use of both KpnI and BglII restriction enzymes. These sites were utilized when constructing pMM-CEN. Building upon the pMM cloning platform, a full length ARS1-CEN4 DNA fragment with compatible ends derived by PCR from plasmid pYAC3 (12) was inserted between KpnI and BglII sites. The ARS1-CEN4 fragment was obtained using ARS-CEN4 primer set (Table 1) and ligated using a standard ligation protocol. Because only one pair of convenient restriction sites was used for cloning of the ARS-CEN4 region, we decided to add a polylinker with the unique sites PacI, XmaI, KasI and insert it into the KpnI site. The resulting construct was verified by PCR using primers specific for kanMX4, hphMX4, ARS-CEN4 and combinations of these genes. Additionally, restriction analysis was performed using the same enzymes as for pMM. Final validation was obtained by transformation of bacteria and yeast followed by selection on media containing ampicillin, kanamycin, hygromycin B in different combinations (for bacteria) and G418 and hygromycin B for yeast.

\section{Transforming Saccharomyces cerevisiae}

Saccharomyces cerevisiae San1 cells were transformed with the standard procedure described by Burke et al. (9) with the slight modification of allowing cells to grow longer (1.5 h in YPD) under non-selective conditions before they were plated on selective medium to give them a better chance to express all resistance genes. Bacterial transformation was performed according to commonly known procedures (7).

\section{Antibiotic resistance test}

Bacterial colonies were picked and grown overnight in LB both supplemented with $100 \mu \mathrm{g} / \mathrm{mL}$ of ampicillin. The following day, $5 \mu \mathrm{L}$ of every overnight culture were inoculated into the same medium. Cultures were grown 6 $\mathrm{h}$ at $37^{\circ} \mathrm{C}$ and then $5 \mathrm{~mL}$ of fresh selective medium containing one or a combination of two or three antibiotics 
Table 1. Primers used for plasmid construction, copy number determination and the translocation cassette production. Relevant restriction sites in primer sequences are written in italic and underlined

Plasmid construction and verification

\begin{tabular}{lc}
\hline Primer sequence & \multicolumn{1}{c}{ Description } \\
\hline F: CGGGATCCCGGACATGGAGGCCCAGAATACCCTCCT & \\
R: GAAGATCTTCGGGGTACCCCCAGTATAGCGACCAGCATTC & BamHI-Hyg ${ }^{R}$-BglII-KpnI set \\
F: GAAGATCTTCAAGCTGTCAAACATGAGAATCTG & \\
R: GGGGTACCCCTTTATTCCCTTGTTGATTC & ARS-CEN4 from pYAC3 set \\
F: ATGGGTAAAAAGCCTGAACTC & \\
R: GAAAAACTCATCGAGCATCA & Verification of pMM and pMM-CEN \\
\hline
\end{tabular}

\begin{tabular}{lc}
\hline & Plasmid copy number determination \\
\hline F: CTACGATACGGGAGGGCTTA & \\
R: ATAAATCTGGAGCCGGTGAG & bla primer set \\
F: CGAGAAACTGGCGATCCTTA & $d x$ primer set \\
R: CTTCATCAAGCGGTTTCACA & \\
\hline
\end{tabular}

Translocation cassette amplification

\begin{tabular}{|c|c|c|c|}
\hline & \multirow{2}{*}{ Cassette type } & \multicolumn{2}{|c|}{ Amplicon size/bp } \\
\hline & & $\mathrm{pMM}$ & pMM-CEN \\
\hline $\begin{array}{l}\text { F: TGGCCCAGCTCATGTTTGACAGC } \\
\text { R: GCGGCCGCATAGGCCACTA }\end{array}$ & $K_{a n}^{R}$ & 1519 & \\
\hline $\begin{array}{l}\text { F: GGACATATTGTCGTTAGAACGCGGC } \\
\text { R: CCCCCAGTATAGCGACCAGCA }\end{array}$ & $H y g^{R}$ & 1710 & \\
\hline $\begin{array}{l}\text { F: GGACATATTGTCGTTAGAACGCGGC+homology ChrXV (160605-160667) } \\
\text { R: GCGGCCGCATAGGCCACT+homology ChrIX (36677-36749) }\end{array}$ & $H y g^{R}-K a n^{R}$ & 3283 & 5256 \\
\hline
\end{tabular}

Translocation verification

Sequence
F: ATTAACGACAAAGACAGCACC
R: GTTAGCTCTAACGTATCTGGTA
R: AATGTCAAGCACTTCCGGAA
F: ATAGGGGCTTAGCATCCACA
R: CCTGGACGTGGGGTCGATTA
F: ATTTTATCCGTACTCCTGATGATGC

Product

\begin{tabular}{ll}
\hline \multicolumn{2}{c}{ Probe synthesis } \\
\hline F: GGTGCGACAATCTATCGATTG & Kanamycin-specific probe synthesis \\
R: AGAAATCACCATGAGTGACGA & \\
F: ATGGGTAAAAAGCCTGAACTC & Hygromycin-specific probe synthesis \\
R: AGATGTTGGCGACCTCGTAT & \\
\hline
\end{tabular}

were inoculated with $10 \mu \mathrm{L}$ of these cultures and grown overnight. The following day, the absorbance was measured at $660 \mathrm{~nm}$ using a GeneQuant ${ }^{\mathrm{TM}}$ pro spectrophotometer (GE Healthcare, Little Chalfont, UK).

LB plates supplemented with increasing concentrations of the antibiotics were used to assess the minimum inhibitory concentration (MIC) of the drugs. pMM-containing E. coli $\mathrm{DH} 5 \alpha$ strains were examined for susceptibility to ampicillin, kanamycin and hygromycin B. The strains were transformed with appropriate plasmids for the experiments: pFA6a-kanMX4 to determine resistance to kanamycin, pUC18 to ampicillin and pAG32 to hygromycin B. Wild type strain was used as a control. Final cell counts were adjusted to $10^{3}, 10^{4}, 10^{5}$ and $10^{6} \mathrm{CFU}$ per $\mathrm{mL}$ of fresh culture per plate. After $24 \mathrm{~h}$ of incubation at $37^{\circ} \mathrm{C}$, microbial growth was checked to determine the MIC value of the various antibiotics.

\section{Plasmid copy number determination}

Plasmid copy number was determined using an approach adapted from a previous report (13). First, an internal standard plasmid was constructed using the pGEM $^{\circledR}-\mathrm{T}$ Easy vector system (Promega). The internal standard plasmid contained both, one gene characteristic of the plasmid to be titrated (bla gene) and one specific for the bacterial chromosome $(d x s)$. The relative primer sets are specified in Table 1. A PCR mixture containing Kapa HiFi polymerase (Kapa Biosystems) and two sets of primers was amplified twice at the limiting number of 20 and 
21 cycles using a Thermo Hybaid Sprint PCR thermal cycler (Ashford, Middlesex, UK). For the internal standard plasmid, the PCR reaction yielded two bands, which were subsequently subjected to densitometric analysis using a Pharmacia-LKB (Uppsala, Sweden) laser scanner densitometer. The intensity of each band was measured three times, and the average value was used to calculate the intensity ratio, which was normalized to 1 . For each measurement, two independent references were used $(1$ and 2 ) and as an internal control, the copy number of reference 2 was determined using the values of reference 1 and vice versa. When PCR amplification was carried out on genomic DNA of cells containing a plasmid, the reaction also yielded two bands: a plasmid-borne bla fragment and a chromosomal $d x$ s. Calculating their ratio and comparing it to the normalized value of the internal standard, the relative plasmid copy number per cell was calculated. For each sample, references 1 and 2 were used, and the average value was accepted. In total, these measurements were repeated 12 times, every time using two internal standards and those from samples 1 to 3 .

\section{Bacterial transformation efficiency}

Both of the constructed plasmids, pMM and pMM-CEN, were used to transform E. coli DH5 $\alpha$ strain and the transformants were selected on ampicillin, kanamycin, hygromycin B, ampicillin-kanamycin, ampicillin-hygromycin B, kanamycin-hygromycin B and ampicillin-kanamycin-hygromycin B plates $(100 \mu \mathrm{g} / \mathrm{mL}$ of each antibiotic). After incubation, number of CFU was determined using a colony counter (Gallenkamp Co, Ltd, London, UK). The transformation efficiency was calculated as the number of transformants per $\mu \mathrm{g}$ of DNA.

\section{Bridge-induced translocations}

Bridge-induced translocation (BIT) is a system that allows to produce chromosomal translocations between any two genomic loci of choice on different chromosomes by means of a PCR-derived DNA bridge called translocation cassette. This is a fragment of DNA where a resistance gene is flanked by two short regions (usually around $64 \mathrm{bp}$ ), each homologous to its chromosomal target. pMM and pMM-CEN offer the possibility, as DNA templates, to amplify cassettes with kanamycin, hygromycin and kanamycin-hygromycin on the same amplicon. In addition, pMM-CEN allows the amplification of a DNA cassette harbouring the CEN4 and an ARS between the kanamycin and hphMX4 resistance genes, thus allowing to test its capacity of bridging two chromosomal portions that do not carry any centromere, a would-be novelty for the BIT system. The pMM plasmid was used as a template for amplification of the BIT translocation cassette aimed to bridge $A D H 1$ locus localized on chromosome XV and SUC2 locus on chromosome IX in S. cerevisiae as described previously (5). Translocation cassettes were produced using Kapa HiFi polymerase (Kapa Biosystems), which contained the two genes conferring resistance to kanamycin and hygromycin B and were used to transform S. cerevisiae San1 strain. Transformants were initially tested on YPD plates containing G418 and hygromycin B. Recombination of the DNA cassette ends into each involved chromosome was tested by colony PCR using the mixture of three primers (Table 1), two localized on the genomic sequences and one localized on the translocation cassette. In the case of chromosome translocation, a product of chromosomal primer and cassette primer was visible (two bands). Detected translocants were examined by CHEF (contour-clamped homogeneous electric field) analysis as described by Tosato et al. (5) and subsequently by Southern blotting with the digoxigenin-labelled probes (DIG probe synthesis kit, Roche, Basel, Switzerland) for kanamycin and $h p h M X 4$ resistance genes to verify the presence of an aberrant chromosome. Primers used for probe synthesis are shown in Table 1.

\section{Results and Discussion}

\section{Construct structure}

Here we report the genetic organization and restriction map of two novel, multidrug resistant marker plasmids: pMM (5581 bp) (Fig. 1) and pMM-CEN (7556 bp) (Fig. 2).

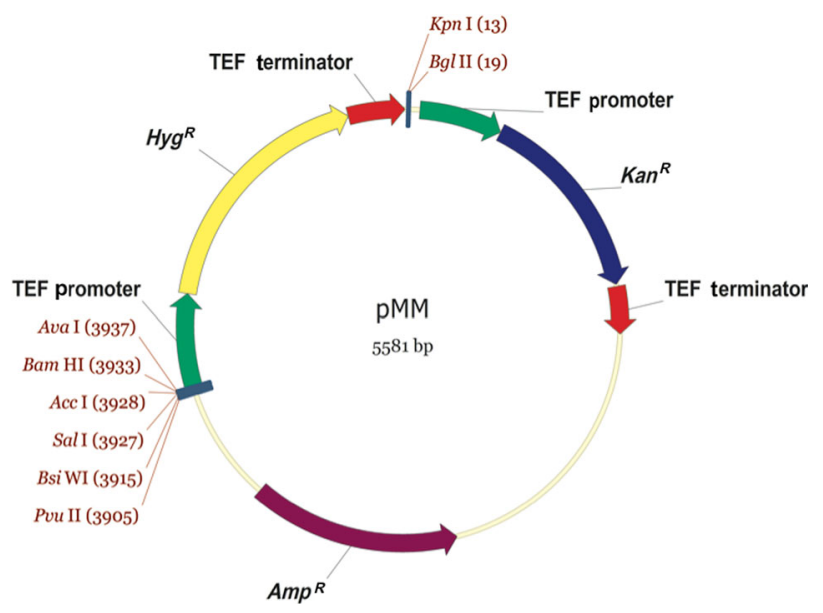

Fig. 1. pMM vector map with unique restriction sites. This construct contains functional cassettes conferring resistance to hygromycin $\left(H y g^{R}\right)$, kanamycin $\left(\operatorname{Kan}^{R}\right)$, and ampicillin $\left(A m p^{R}\right)$. Both, kanamycin and hygromycin are under control of the translation elongation factor (TEF) promoter and terminator. Numbers in brachets represent the position of the restriction sites

These plasmid constructs contain four important genetic elements placed on the same DNA molecule: a dominant resistance gene for ampicillin (bla), kanamycin (kanMX4 in bacteria) and geneticin (G418 in yeast), hygromycin (hphMX4) (11), and an ARS-CEN4 DNA module (only for PMM-CEN) providing stable maintenance in the yeast cells. The kanMX4 is a DNA segment containing the open reading frame of aminoglycoside phosphotransferase from E. coli transposon Tn903 and the TEF1 (translation elongation factor) promoter and terminator derived from the filamentous fungus Ashbya gossypii, providing transcriptional and translational control $(10,14)$. Expression of kanMX4 renders bacteria resistant to kanamycin and yeast cells resistant to geneticin (G418) $(15,16)$. The presence of the $h p h M X 4$ gene confers resistance to the antibiotic hygromycin B (11) and the coding region of this 


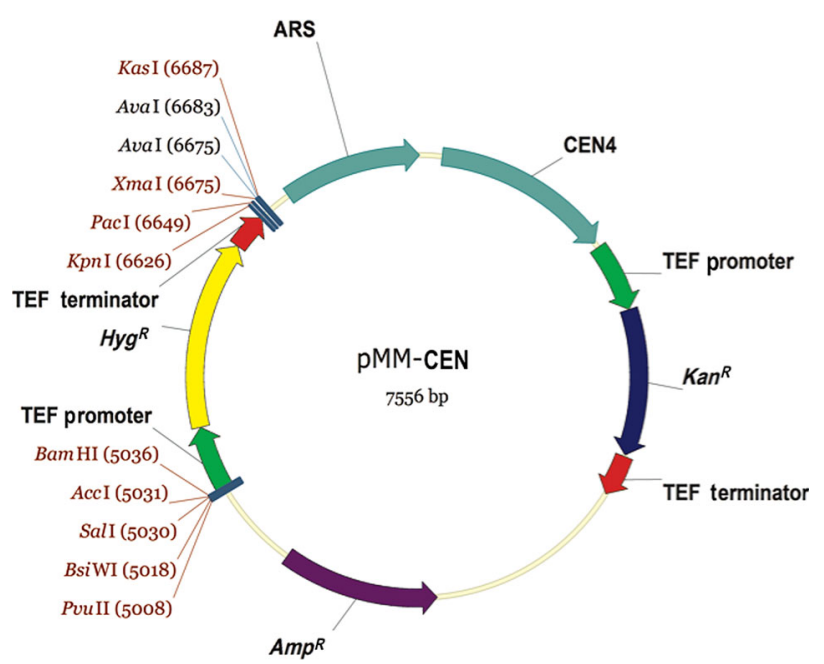

Fig. 2. pMM-CEN vector map with unique restriction sites. This vector can be used as a shuttle vector due to the presence of CEN4 and ARS elements providing stable maintenance in the buddying yeast. Analogously to the previous vector, this construct confers resistance to kanamycin $\left(\mathrm{Kan}^{R}\right)$ and hygromycin $\left(H y g^{R}\right)$ thanks to the cassettes present within its structure. Moreover, for selection in the bacterial hosts, ampicillin might be used due to the presence of the $A m p^{R}$ gene. TEF=translation elongation factor. Numbers in brachets represent the position of the restriction sites

gene is also under the transcriptional and translational control of the TEF1 promoter and terminator. The correct presence of these elements was confirmed by colony PCRs, as explained in Materials and Methods. For construction of pMM-CEN, the full-length ARS-CEN4 region from the pYAC3 plasmid was inserted between the KpnI and BglII sites. The lack of convenient restriction sites between the markers on pMM-CEN prompted us to add a polylinker fragment in the KpnI site carrying PacI, XmaI and KasI sites. PacI leaves 3 ' overhang, whereas Xmal and KasI leave 5' overhang, successfully preventing self-ligation. Additionally, they work in the same buffers and do not exhibit star activity, which makes working with them very simple. These cloning sites can be utilized in several different ways, allowing construction of two-marker cassettes separated by any DNA sequence or two-marker cassette with a centromeric sequence. Suggested primers for cassette amplification are shown in Table 1.

These primers were practically tested on the pMM-CEN template and their PCR products showed the correct size (not shown).

\section{Antibiotic resistance tests}

Resistance tests indicate that both yeast and bacterial cells were rendered resistant to kanamycin and hygromycin B by the introduction of the pMM plasmid. These two drugs belong to the same group of aminoglycosidic antibiotics and have a similar mode of action (17-21), which helps to reduce the incidence of spontaneous resistance and lowers the percentage of false positives. Additionally, the plasmid confers resistance to ampicillin thanks to the presence of the b-lactamase gene, thus providing additional means for selection in bacteria $(22,23)$. When bacterial cells were transformed with pMM, they exhibited re- sistance to each of the drugs separately, as well as to all combinations of these agents (ampicillin-kanamycin, ampicillin-hygromycin B, kanamycin-hygromycin B and ampicillin-kanamycin-hygromycin B), rendering this system quite flexible for the selection of multiple resistances. The same was observed in the case of yeast transformation, where the presence of pMM renders cells able to grow on geneticin- and/or hygromycin B-containing plates $(200 \mu \mathrm{g} / \mathrm{mL}$ of $\mathrm{G} 418$ and $300 \mu \mathrm{g} / \mathrm{mL}$ of hygromycin B, respectively). Transformation with the second plasmid construct, pMM-CEN, confirmed these results. However, pMM-containing yeast colonies were, as expected, smaller than pMM-CEN, due to the lack of stable maintenance and transmission of the plasmid within the population. We tested the range of plasmid-mediated resistance on each of the drugs separately and in combinations with each other and determined transformation efficiency.

The minimum inhibitory concentration (MIC) was tested with E. coli $\mathrm{DH} 5 \alpha$ strains transformed with different plasmids by their ability to produce visible growth on a series of agar plates that contain increasing concentrations of three antibiotics. The concentration of ampicillin on LB agar plates was between 50 and $100 \mu \mathrm{g} / \mathrm{mL}$, of kanamycin was between 100 and $5000 \mu \mathrm{g} / \mathrm{mL}$, and of hygromycin B between 100 and $5000 \mu \mathrm{g} / \mathrm{mL}$.

As shown in Fig. 3, the range of plasmid-mediated resistance to each of the drugs was tested separately and compared with parental plasmid resistance (see Materials and Methods). In the case of ampicillin, all tested bacterial clones were surprisingly resistant up to $10000 \mu \mathrm{g} / \mathrm{mL}$. When tested on kanamycin, cells containing pFA6a-kanMX4 and pMM exhibited resistance up to $1000 \mu \mathrm{g} / \mathrm{mL}$ and no fluctuations of the values compared to the parental plasmid were observed. A different situation was noticed in the hygromycin B resistance test, in which pMM-containing cells grew in a medium with $2000 \mu \mathrm{g} / \mathrm{mL}$ of the drug, while the parental plasmid pAG32 carrying the resistance gene $p h p M X 4$ exhibited only slight growth in $1000 \mu \mathrm{g} / \mathrm{mL}$ of hygromycin B medium. One possible explanation of this phenomenon could be that the phpMX4 promoter on $\mathrm{pMM}$ plasmid is subject to a greater rate of activation than when located on pAG32.

\section{Bacterial transformation efficiency and selection of different markers}

Since bacteria harbouring one of our newly constructed plasmids should exhibit resistance to the three markers separately, as well as in mixed configurations, we transformed bacterial cells with pMM and pMM-CEN and selected on ampicillin, kanamycin, hygromycin B and the combination of ampicillin-kanamycin, ampicillin-hygromycin B, kanamycin-hygromycin B and ampicillin-kanamycin-hygromycin B plates. Each transformation was repeated 3 times using the same aliquot of pMM and pMM-CEN miniprep. To visualize the influence of single, double and triple selection on the number of transformants, transformation efficiencies were calculated of both plasmids when growing on media containing different combinations of the drugs. The results (Table 2) showed that the highest transformation efficiency of both plasmids was observed when single selection was applied. These values dropped as expected when two drugs were 


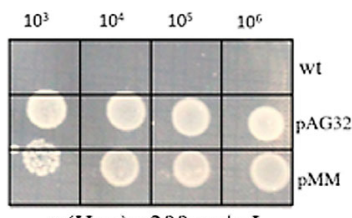

$\gamma(\mathrm{Hyg})=200 \mu \mathrm{g} / \mathrm{mL}$
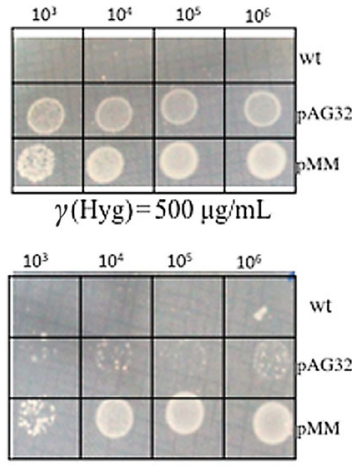

$\gamma(\mathrm{Hyg})=1000 \mu \mathrm{g} / \mathrm{mL}$

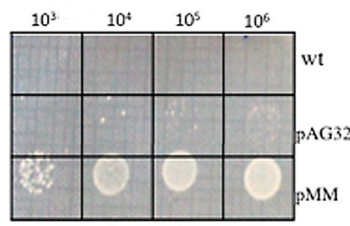

$\gamma(\mathrm{Hyg})=2000 \mu \mathrm{gg} / \mathrm{mL}$

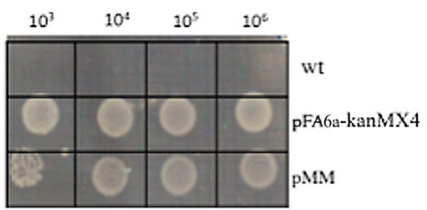

$\gamma(\mathrm{Kan})=200 \mu \mathrm{g} / \mathrm{mL}$

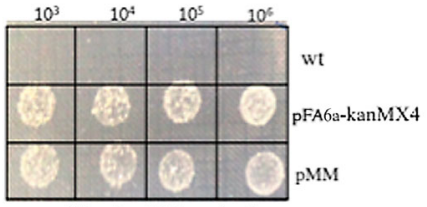

$\gamma(\mathrm{Kan})=500 \mu \mathrm{g} / \mathrm{mL}$

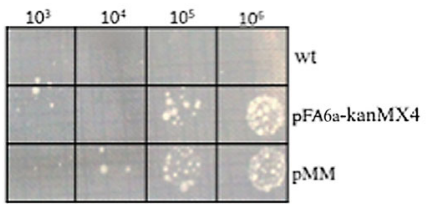

$\gamma(\mathrm{Kan})=1000 \mu \mathrm{g} / \mathrm{mL}$

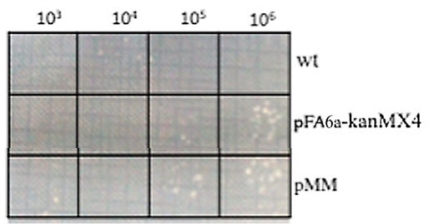

$\gamma(\mathrm{Kan})=2000 \mu \mathrm{g} / \mathrm{mL}$

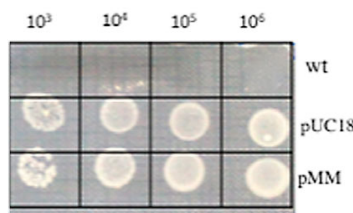

$\gamma($ Amp $)=500 \mu \mathrm{g} / \mathrm{mL}$

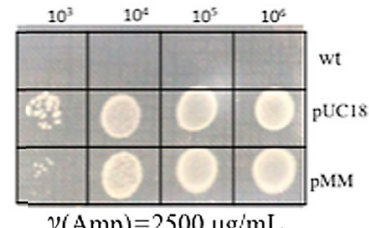

$\gamma($ Amp $)=2500 \mu \mathrm{g} / \mathrm{mL}$

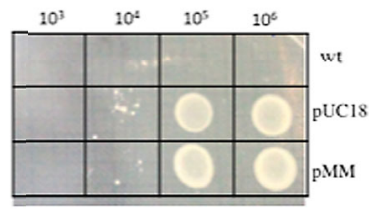

$\gamma(\mathrm{Amp})=5000 \mu \mathrm{g} / \mathrm{mL}$

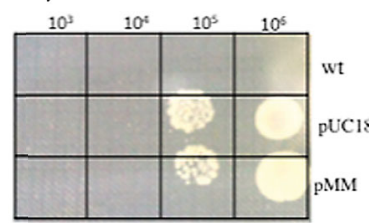

$\gamma($ Amp $)=10000 \mu \mathrm{g} / \mathrm{mL}$

Fig. 3. Determination of minimum inibitory concentration (MIC) values with pMM-containing cells on each of the different concentrations of drugs. E. coli DH5 $\mu$ strains were used for the experiments with the appropriate plasmids: pFA6a-kanMX4 for kanamycin, pUC18 for ampicillin and pAG32 for hygromycin B resistance. The spots represent growth of different dilutions of a cell culture starting with approx. $10^{3}$ cells $/ \mathrm{mL}$ from left to right. Amp=ampicillin, Hyg=hygromycin, Kan=kanamycin, wt=wild type strains

Table 2. Transformation efficiency values of plasmids pMM and pMM-CEN determined for different combinations of antibiotics

\begin{tabular}{llccccccc}
\hline & & Amp & Kan & Hyg & Amp-Kan & Kan-Hyg & Amp-Hyg & $\begin{array}{c}\text { Amp- } \\
\text {-Kan-Hyg }\end{array}$ \\
\hline \multirow{2}{*}{ pMM } & Average transformation efficiency & $1.81 \cdot 10^{4}$ & $1.29 \cdot 10^{4}$ & $1.66 \cdot 10^{4}$ & $0.95 \cdot 10^{4}$ & $1.28 \cdot 10^{4}$ & $1.31 \cdot 10^{4}$ & $0.44 \cdot 10^{4}$ \\
& SEM & $0.12 \cdot 10^{4}$ & $0.09 \cdot 10^{4}$ & $0.11 \cdot 10^{4}$ & $0.90 \cdot 10^{4}$ & $0.10 \cdot 10^{4}$ & $0.07 \cdot 10^{4}$ & $0.06 \cdot 10^{4}$ \\
\hline \multirow{2}{*}{ pMM- } & Average transformation efficiency & $0.82 \cdot 10^{4}$ & $0.65 \cdot 10^{4}$ & $0.70 \cdot 10^{4}$ & $0.11 \cdot 10^{4}$ & $0.16 \cdot 10^{4}$ & $0.42 \cdot 10^{4}$ & $0.08 \cdot 10^{4}$ \\
-CEN & SEM & $1.81 \cdot 10^{4}$ & $1.29 \cdot 10^{4}$ & $1.66 \cdot 10^{4}$ & $0.02 \cdot 10^{4}$ & $0.02 \cdot 10^{4}$ & $0.01 \cdot 10^{4}$ & $0.01 \cdot 10^{4}$ \\
\hline
\end{tabular}

Amp=ampicillin, Kan=kanamycin, Hyg=hygromycin, SEM=standard error of the mean $(N=3)$

used, with the most toxic being ampicillin-kanamycin. When three markers were combined, the transformation efficiency was reduced five- (pMM) and tenfold (pMM-CEN). Triple selection allowed only triple-resistant cells to divide and potentially eliminate all background of false transformants.

On the other hand, since both $\mathrm{Kan}^{R}$ and $H y g^{R}$ resistance genes share the same TEF1 promoter/terminator elements, recombination between their copies cannot be excluded, although a $\mathrm{rec}^{-}$strain was used. In this case, recombination between two direct DNA repeats represented by the TEF1 promoter sequences on plasmid pMM would lead to the excision of the hphMX4 resistance gene, while recombination between the two TEF1 terminators would lead to excision and loss of the $\mathrm{Kan}^{R}$ marker. Therefore, what could have been interpreted as background, i.e. the loss of a resistance phenotype, could very well be due to the loss of a portion of the plasmid carrying that resi- stance gene, which underwent recombination, rather than a classical background due to spontaneous mutation.

\section{Plasmid copy number}

The observed high levels of antibiotic resistance could suggest that plasmids exist in high copy numbers in bacterial cells (in yeast cells, pMM-CEN number is limited to one or two due to the presence of centromeric sequences), while the control plasmids, pAG32 and pFA6a-kanMX4 showed approx. 15-20 copies per cell (data not shown) and pUC18 is reported to reach a very high copy number of more than $500(24,25)$. To our surprise, after combining data from 12 independent experiments, we observed that copy number is rather moderate and oscillates around 10 copies per cell $(10.77 \pm 1.57)$. Moreover, these plasmids can be stably maintained for almost twenty generations in bacteria. 


\section{The pMM plasmid as a useful template for the production of BIT DNA cassettes}

As an experimental application of these new vectors, the pMM plasmid was employed for the amplification of DNA cassettes used for bridge-induced chromosome translocation (BIT) between the two yeast loci $A D H 1$ on chromosome XV and SUC2 on chromosome IX (Fig. 4). We successfully produced a $\mathrm{Kan}^{R}-H y g^{R}$ DNA cassette by PCR on the PMM template, utilizing the primers described in Table 1. This DNA cassette was then used to transform yeast to induce translocations between chromosomes IX and XV.

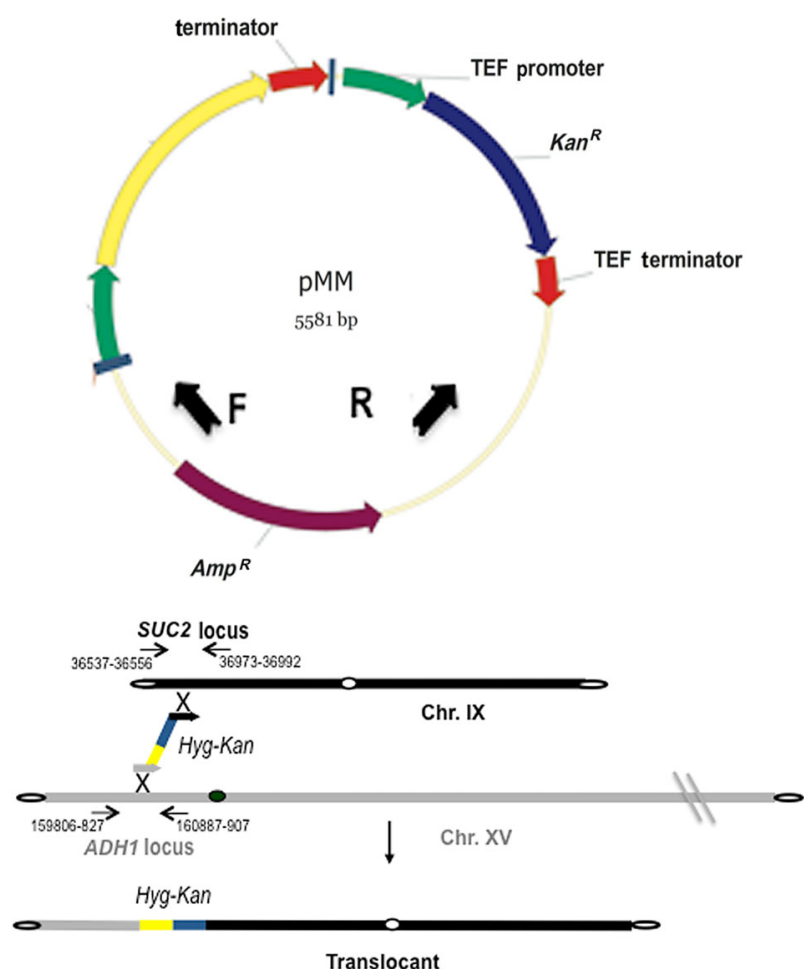

Fig. 4. At the top of the figure, the PCR amplification of the cassette carrying the $\mathrm{Kan}^{R}-\mathrm{Hyg}^{R}$ genes using the pMM template is shown. The primers used, named F (forward) and R (reverse; sequences in Table 1), are drawn in the figure as thick black arrows. At the bottom, the scheme of the translocation between chromosomes XV and IX is represented. The primers used to check the correct integration and their relative position are indicated by thin arrows with sequence numbers. The two break lines on chromosome XIV indicate that the drawing of the chromosomes is not proportional

After colony PCR screen, we were able to find translocants resistant to kanamycin and hygromycin B simultaneously. The stability of pMM-derived cassette was confirmed by a CHEF gel when compared to the wild type San1 strain (not shown). Moreover, Southern hybridization revealed strong signal when kanamycin- or hygromycin B-specific probe was used and this is a strong evidence that translocation cassette gets integrated into selected loci and is not lost during cell division. Verification experiments are summarized in Fig. 5. This demonstrated a successful application of this new vector and the relative in vivo stability of the two markers in a recombination proficient yeast strain.

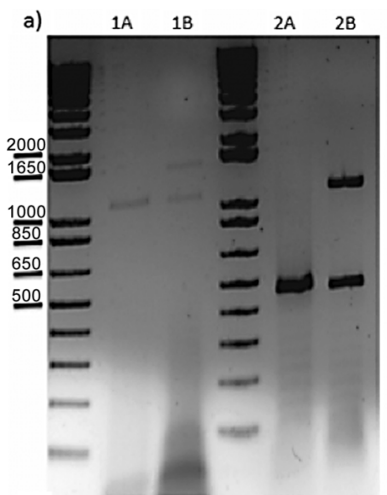

c)

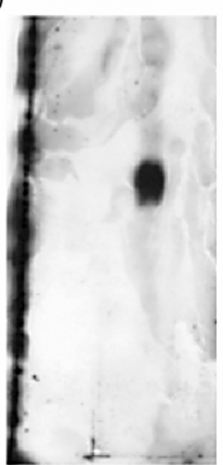

b) 12

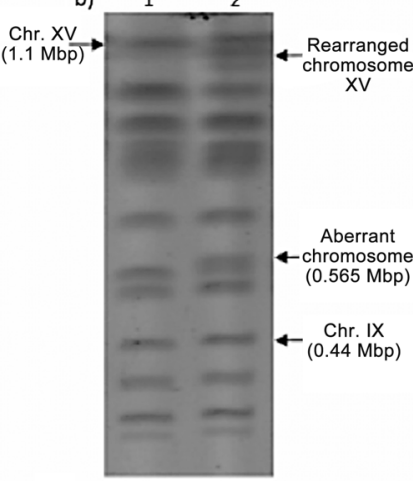

d)

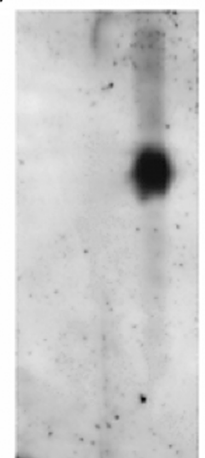

Fig. 5. a) Colony PCR verification of translocants obtained with $K{ }^{R}-H y g^{R}$ cassette: lane 1A: ADH1 band obtained on San1 strain. Lane 1B: PCR product obtained from San1 strain carrying the translocated chromosome. The upper band reveals integration of the cassette into chromosome $\mathrm{XV}$, while the lower band is the ADH1 wild type (wt) band. Analogously, lanes 2A and 2B contain colony PCR products on the wt and translocant strain for the second translocation point (SUC2 on chromosome IX); b) CHEF gel electrophoresis of translocation involving chromosomes XV and IX. Lane 1: wt San1 strain, lane 2: San1 translocant; c) and d) Southern hybridization with DIG probe specific for kanamycin and hygromycin respectively, providing direct evidence of translocation cassette integration into the buddying yeast genome

The added value of this vector with respect to a single-marker one is that, at the onset of selection after transformation, one can immediately apply a single (resistance to kanamycin or hygromycin B) or double (resistance to both) selection, thereby imposing a much flexible and restrictive selective pressure on the cells that have uptaken the transforming DNA plasmid. According to our previous experience with BIT, this vector significantly improved the selection efficiency, as it was possible to select on much higher doses of one antibiotic, or on two antibiotics, thereby reducing the false-positive background by an estimated 35 or $55 \%$, respectively. When we performed BIT with a DNA cassette amplified on the pMM template, we measured the rate of excision between TEF regions by counting cells that lost resistance to one or the other drug, due to direct repeat recombination. The ratio was rather low, as out of 800 yeast colonies originally resistant to both drugs, $62(7.75 \%)$ exhibited excision of the $\mathrm{Kan}^{R}$ marker, and $30(3.75 \%)$ of the $H y g^{R}$.

Recombination between TEF elements on pMM-CEN should also lead to the exclusion of the centromere CEN and ARS elements, thus this plasmid would be quickly 
lost in the population. Furthermore, the inclusion of the ARS-CEN DNA, encoding a DNA sequence for the yeast origin of replication and the centromere, would allow the recovery of translocations connecting two portions of chromosomes devoided of them.

S. cerevisiae is a very important organism employed during various genetic analyses in which gene disruption, deletion, tagging, or cloning are the main part of the whole experimental assay. Gene cloning in yeast requires convenient shuttle plasmids (integrative, centromeric or episomal) like the commonly used vectors of the pRS series (26) and their further modifications (26-28). In addition, genome manipulations such as gene deletions or replacements are usually based on drug resistance markers used for gene disruption or tagging $(29,30)$ and require convenient PCR templates for amplification of deletion cassettes. The majority of the vectors present on the market offer the possibility of selection by the means of auxotrophic markers. However, this is not always convenient, as frequently the strain is marker-incompatible (which requires additional manipulation of the genome and generation of designer strains $(31,32))$. Moreover, in particular approaches, the experimental set up might require growth in rich medium, which renders auxotrophic selection impossible. Transformation-associated background can be another condition rendering some experiments very difficult. A similar system, where the URA3 marker was replaced by the kanMX4 module has been constructed, but until now no double antibiotic selection marker has been available (33). In this work, we observed simultaneous resistance up to two (in the case of yeast) and three (in bacteria) antibiotic agents. Another interesting and important result is that reported in Fig. 3, where the growth tests demonstrated a superior resistance of bacteria to a particular antibiotic when all three, ampicillin, kanamycin and hygromycin B resistance genes were present and expressed on the same plasmid carrier. Although the nature of the metabolic interaction leading to this phenomenon is not clear, its role in the understanding of spontaneous acquisition of multiple antibiotic resistance must be considered. This result warrants an analogous investigation in yeast to verify the possible extension of the phenomenon to eukaryotic model cell system as well.

\section{Conclusions}

Our two newly produced plasmid constructs, pMM and $\mathrm{pMM}-\mathrm{CEN}$, carry features making them very handy and valuable tools for all modern molecular biology tasks, especially in functional analyses, recombinant DNA work and cloning experiments. The newly constructed pMM and pMM-CEN can be used for these approaches and possess the advantage of permitting the amplification of one, two or three antibiotics on the same DNA cassette, over commonly used vectors. Moreover, pMM-CEN allows the inclusion of a centromere DNA sequence able to stabilize the newly formed transforming chromosome lacking its own centromere DNA sequence. These plasmids can be used completely independently from the auxotrophic markers in the host strain, allowing their utilization in natural yeast isolates where strains were heavily manipulated and no auxotrophic selection marker is available.
Our initial goal was to build a multi-marker plasmid system, believing that this could be used readily as it is, while further applications have proven its additional value as a convenient platform for further genomic modifications. The experimental results indicated that clones carrying these constructs could be subjected to very stringent selective conditions like the triple selection that can be successfully used facilitating the limitation of an unwanted background. In order to validate the pMM plasmid ability to be used as a PCR template for genome manipulation, we used it as a template for amplifying a chromosome translocation DNA cassette used subsequently in the BIT procedure. As expected, the cassette successfully induced translocation between the ADH1 and SUC2 loci. The resulting clones carried the correct translocated chromosome and exhibited resistance to kanamycin and hygromycin B drugs separately and in combination. The use of double selection lowered the background, probably by allowing only the most fit clones to survive. Another issue when working with yeast shuttle plasmids is the lack of convenient restriction sites for cloning. To expand more the versatility of pMM-CEN, a polylinker containing unique restriction sites was added. In addition to the ability of this plasmid to be stably maintained and transmitted through generations, the presence of these cloning sites will greatly facilitate the work with this vector.

\section{Aknowledgements}

R. Arnak was a PhD student supported by an ICGEB pre-doctoral fellowship; B. Altun was a PhD student supported by the Turkish Government and by the TRIL (Training and Research in Italian Laboratories) program.

\section{References}

1. Casali N, Preston A, editors. E. coli plasmid vectors: methods and applications. New York, NY, USA: Humana Press; 2003.

2. Walsh C. Antibiotics: actions, origins, resistance. Washington DC, USA: ASM Press; 2003.

3. Baudin A, Ozier-Kalogeropoulos O, Denouel A, Lacroute F, Cullin C. A simple and efficient method for direct gene deletion in Saccharomyces cerevisiae. Nucleic Acids Res. 1993;21: 3329-30. http://dx.doi.org/10.1093/nar/21.14.332915

4. Tosato V, Nicolini C, Bruschi CV. DNA bridging of yeast chromosomes VIII leads to near-reciprocal translocation and loss of heterozygosity with minor cellular defects. Chromosoma. 2009;118:179-91.

http://dx.doi.org/10.1007/s00412-008-0187-z

5. Tosato V, Waghmare SK, Bruschi CV. Non-reciprocal chromosomal bridge-induced translocation (BIT) by targeted DNA integration in yeast. Chromosoma. 2005;114:15-27. http://dx.doi.org/10.1007/s00412-005-0332-x

6. Mahamoud A, Chevalier J, Alibert-Franco S, Kern WV, Pagès JM. Antibiotic efflux pumps in Gram-negative bacteria: the inhibitor response strategy. J Antimicrob Chemother. 2007; 59:1223-9. http://dx.doi.org/10.1093/jac/dkl493

7. Maniatis T, Fritsch EF, Sambrook J. Molecular cloning: a laboratory manual. Cold Spring Harbor, NY, USA: Cold Spring Harbor Lab Press; 1982.

8. Waghmare SK, Caputo V, Radovic S, Bruschi CV. Specific targeted integration of kanamycin resistance-associated nonse- 
lectable DNA in the genome of the yeast Saccharomyces cerevisiae. Biotechniques. 2003;34:1024-8.

9. Burke D, Dawson D, Stearns T. Methods in yeast genetics: a Cold Spring Harbor Laboratory course manual. Cold Spring Harbor, NY, USA: Cold Spring Harbor Lab Press; 2000.

10. Wach A, Brachat A, Pöhlmann R, Philippsen P. New heterologous modules for classical or PCR-based gene disruptions in Saccharomyces cerevisiae. Yeast. 1994;10:1793-808.

11. Goldstein AL, McCusker JH. Three new dominant drug resistance cassettes for gene disruption in Saccharomyces cerevisiae. Yeast. 1999;15:1541-53. http://dx.doi.org/10.1002/(SICI)1097-0061(199910)15:14<1541: :AID-YEA476>3.0.CO;2-K

12. Burke DT, Carle GF, Olson MV. Cloning of large segments of exogenous DNA into yeast by means of artificial chromosome vectors. Science. 1987;236:806-12. http://dx.doi.org/10.1126/science.3033825

13. Lee C, Kim J, Shin SG, Hwang S. Absolute and relative QPCR quantification of plasmid copy number in Escherichia coli. J Biotechnol. 2006;123:273-80.

14. Wach A. PCR-synthesis of marker cassettes with long flanking homology regions for gene disruptions in S. cerevisiae. Yeast. 1996;12:259-65.

15. Davies J, Jimenez A. A new selective agent for eukaryotic cloning vectors. Am J Trop Med Hyg. 1980;29:1089-92.

16. Hadfield C, Jordan BE, Mount RC, Pretorius GH, Burak E. G418-resistance as a dominant marker and reporter for gene expression in Saccharomyces cerevisiae. Curr Genet. 1990; 18:303-13.

17. Handbook of Anti-Tuberculosis Agents: Kanamycin. Tuberculosis. 2008;88:117-8. http://dx.doi.org/10.1016/S1472-9792(08)70012-X

18. Blochlinger K, Diggelmann H. Hygromycin B phosphotransferase as a selectable marker for DNA transfer experiments with higher eucaryotic cells. Mol Cell Biol. 1984;4:2929-31. http://dx.doi.org/10.1128/MCB.4.12.2929

19. Borovinskaya MA, Shoji S, Fredrick K, Cate JH. Structural basis for hygromycin $\mathrm{B}$ inhibition of protein biosynthesis. RNA. 2008;14:1590-9. http://dx.doi.org/10.1261/rna.1076908

20. Rao RN, Allen NE, Hobbs JN Jr, Alborn WE Jr, Kirst HA, Paschal JW, Genetic and enzymatic basis of hygromycin B resistance in Escherichia coli. Antimicrob Agents Chemother. 1983;24:689-95. http://dx.doi.org/10.1128/AAC.24.5.689

21. Umezawa H. Studies on aminoglycoside antibiotics: enzymic mechanism of resistance and genetics. Jpn J Antibiot. 1979;32:S1-14
22. Donowitz GR, Mandell GL. Beta-Lactam antibiotics (1). N Engl J Med. 1988;318:419-26.

23. Sutcliffe JG. Nucleotide sequence of the ampicillin resistance gene of Escherichia coli plasmid pBR322. Proc Natl Acad Sci USA. 1978;75:3737-41.

24. Yanisch-Perron C, Vieira J, Messing J. Improved M13 phage cloning vectors and host strains: nucleotide sequences of the M13mp18 and pUC19 vectors. Gene. 1985;33:103-19.

25. Lin-Chao S, Chen WT, Wong TT. High copy number of the pUC plasmid results from a Rom/Rop-suppressible point mutation in RNA II. Mol Microbiol. 1992;6:3385-93.

26. Sikorski RS, Hieter P. A system of shuttle vectors and yeast host strains designed for efficient manipulation of DNA in Saccharomyces cerevisiae. Genetics. 1989;122:19-27.

27. Chee MK, Haase SB. New and redesigned pRS plasmid shuttle vectors for genetic manipulation of Saccharomyces cerevisiae. G3 (Bethesda). 2012;2:515-26. http://dx.doi.org/10.1534/g3.111.001917

28. Frazer LN, O'Keefe RT. A new series of yeast shuttle vectors for the recovery and identification of multiple plasmids from Saccharomyces cerevisiae. Yeast. 2007;24:777-89. http://dx.doi.org/10.1002/yea.1509

29. Knop M, Siegers K, Pereira G, Zachariae W, Winsor B, Nasmyth K, Schiebel E. Epitope tagging of yeast genes using a PCR-based strategy: more tags and improved practical routines. Yeast. 1999;15:963-72. http://dx.doi.org/10.1002/(SICI)1097-0061(199907)15:10B<963: :AID-YEA399>3.0.CO;2-W

30. Walker M, Vystavelova A, Pedler S, Eglinton J, Jiranek V. PCR-based gene disruption and recombinatory marker excision to produce modified industrial Saccharomyces cerevisiae without added sequences. J Microbiol Methods. 2005;63: 193-204.

31. Brachmann CB, Davies A, Cost GJ, Caputo E, Li J, Hieter P, Boeke JD. Designer deletion strains derived from Saccharomyces cerevisiae S288C: a useful set of strains and plasmids for PCR-mediated gene disruption and other applications. Yeast. 1998;14:115-32. http://dx.doi.org/10.1002(SICI)1097-0061(19980130)14:2<115: :AID-YEA204>3.0.CO;2-2

32. Replogle K, Hovland L, Rivier DH. Designer deletion and prototrophic strains derived from Saccharomyces cerevisiae W303-1a. Yeast. 1999;15:1141-9. http://dx.doi.org/10.1002/(SICI)1097-0061(199908)15:11<1141: :AID-YEA439>3.0.CO;2-P

33. Bardazzi I, Casalone E. Construction of two new vectors for transformation of laboratory, natural and industrial Saccharomyces cerevisiae strains to trifluoroleucine and G418 resistance. Folia Microbiol (Praha). 2004;49:534-8. 